\title{
Ultrathin cameras using annular folded optics
}

\author{
Eric J. Tremblay, Ronald A. Stack, Rick L. Morrison, and Joseph E. Ford
}

\begin{abstract}
We present a reflective multiple-fold approach to visible imaging for high-resolution, large aperture cameras of significantly reduced thickness. This approach allows for reduced bulk and weight compared with large high-quality camera systems and improved resolution and light collection compared with miniature conventional cameras. An analysis of the properties of multiple-fold imagers is presented along with the design, fabrication, and testing of an eightfold prototype camera. This demonstration camera has a $35 \mathrm{~mm}$ effective focal length, $0.7 \mathrm{NA}$, and $27 \mathrm{~mm}$ effective aperture folded into a $5 \mathrm{~mm}$ total thickness. (C) 2007 Optical Society of America
\end{abstract}

OCIS codes: $\quad 080.3620,110.0110,100.2000$.

\section{Introduction and Background}

Reducing the bulk and weight of visible imaging systems is important for applications where bulky conventional imaging systems are unsuitable, as in surveillance cameras for miniaturized unmanned aerial vehicles and portable infrared telescopes. Although miniature cameras such as those found in cell phones are now commonplace, their resolution and light collection compare poorly with their full size counterparts. Here we investigate the use of multiple-fold reflective optics to design imagers of significantly reduced thickness, high resolution, and large light collection compared with miniature refractive cameras.

Our approach to creating an ultrathin high-quality imager is based on an extension of conventional astronomical telescopes such as the Cassegrain telescope, ${ }^{1}$ with additional folding ${ }^{2,3}$ shown in Fig. 1(b). Light enters the element through an outer annular aperture and is focused by a series of concentric zone reflectors to the image plane in the central area of the optic.

Although the fundamental optical concept used in this technology has existed for several hundred years, it has not found widespread use in compact camera

E. J. Tremblay (etremblay@ucsd.edu) and J. E. Ford are with the Department of Electrical and Computer Engineering, University of California San Diego, La Jolla, California 92093. R. A. Stack and R. L. Morrison are with Distant Focus Corporation, Champaign, Illinois 61820-7460.

Received 13 June 2006; revised 19 September 2006; accepted 21 September 2006; posted 22 September 2006 (Doc. ID 71933); published 17 January 2007.

0003-6935/07/040463-09\$15.00/0

(C) 2007 Optical Society of America systems. It is likely that, when previously considered, the difficulty associated with fabricating a single optical element containing several concentric surfaces of extremely tight relative tolerances eclipsed the benefits gained by doing so. The current demand for lightweight, thin, high-quality imagers for aerospace, consumer, and military applications along with the recent maturity of single-point diamond turning technology now makes this approach advantageous and possible. Diamond turning technology, used primarily for fabricating high-quality IR optics, can now produce a good surface figure for visible light. Further magnetorheological polishing can improve it further. ${ }^{4}$

Wavefront coding is an imaging technique introduced by Dowski and Cathey ${ }^{5}$ utilizing joint optimization of a coded phase plate and digital postdetection processing. Such techniques will be useful in annular folded optic imagers to increase invariance to defocus related aberrations, improve fabrication tolerances and depth of field in a way not possible with traditional optical systems. The implementation of wavefront coding and postprocessing will be addressed in a subsequent paper.

Here we examine the fundamental characteristics of multiple-fold annular imagers and define several of the challenges associated with them. Section 2 discusses multiple-fold imagers in general, covering basic properties, advantages, and challenges of folded design. Section 3 explores the design space, discussing design constraints and considerations regarding our specific design goals. Section 4 covers the design, fabrication, and experimental demonstration of an eightfold camera prototype, and Section 5 contains our conclusions. 


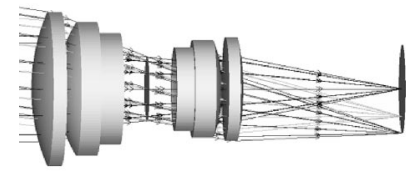

(a)

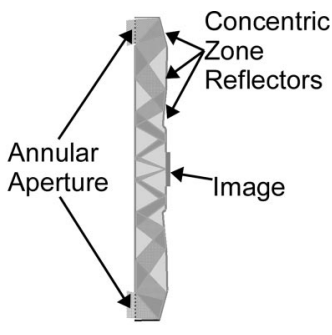

(b)
Fig. 1. (a) Conventional compound refractive lens. (b) Annular folded optics concept.

\section{General Properties of Multiple-Fold Reflective Imagers}

\section{A. Resolution and Light Collection}

Resolution is typically poor in miniature conventional optics since the focal length is reduced without a similar decrease in pixel and array size. Currently, the minimum pixel pitch found to be commercially available is $2.2 \mu \mathrm{m}$ square in complementary metal-oxide semiconductor (CMOS) sensors (Omnivision OV3630) and $2.35 \mu \mathrm{m}$ square in CCD sensors (Sony ICX488DQ/DQF). For miniature cameras, when the physical size constraints limit the focal length of the imager optics, these minimum pixel sizes limit the achievable resolution. Folding the optic enables a longer effective focal length without increasing the optical track (the physical length from first surface to image sensor). This allows for greater magnification and increased angular resolution subtended by the pixel sampling pitch.

In addition to extending the focal length, folding the optic also enlarges the diameter of the imager increasing the collection aperture area compared with miniature conventional cameras of the same thickness. To compare the total optical power collected by the aperture of an annular folded optic with that of a conventional unobscured circular optic, we can define an effective aperture diameter for the folded optic as

$$
d_{\text {eff }}=d_{\text {outer }} \sqrt{1-o^{2}}
$$

where $d_{\text {outer }}$ is the outer diameter (OD) of the folded optic, $o$ is the obscuration ratio (the inner aperture diameter divided by the outer aperture diameter), and $d_{\text {eff }}$ is the diameter of an unobscured circular aperture of the same aperture area as the folded optic. Figure 2 shows this relationship between the $\mathrm{OD}$ and the obscuration to maintain constant optical input power for several effective aperture diameters. For example, a folded optic with an obscuration ratio of $90 \%$ (inner diameter $0.9 \times$ OD) will have an effective diameter $2.29 \times$ smaller than its actual outer diameter. Stated another way, the folded optic diameter will have to be scaled in size by $2.29 \times$ to match the collection area of a conventional optic. Multiplefold optics will typically have aspect ratios (diameter/ thickness) larger than 2 and larger total collection

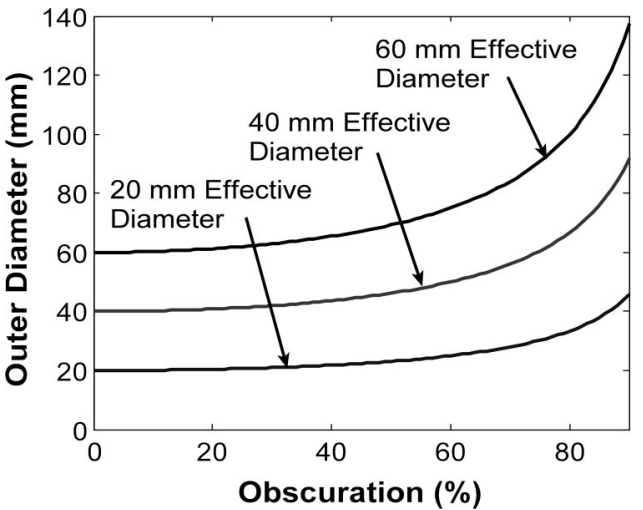

Fig. 2. OD versus obscuration to maintain constant collection aperture area (effective diameter) compared to an unobscured lens.

areas than conventional fast lenses of the same track length.

\section{B. Physical Optics of Annular Apertures}

When the number of concentric folds in an optic is large, diffraction effects from the highly obscured circular aperture must be considered. For a given diameter and focal length, an annular aperture moves optical power in the incoherent point-spread function (PSF) from the central peak into the sidelobes, reducing the midspatial frequency modulation transfer function (MTF). ${ }^{6-8}$ The incoherent PSF for an annular aperture can be expressed analytically by Eq. (2) and shown for various levels of obscuration in Fig. 3(a) ${ }^{9}$ :

$$
\begin{aligned}
I(r)= & \left(\frac{1}{\lambda z}\right)^{2} \mid\left(\pi a_{2}^{2}\right)\left[\frac{2 J_{1}\left(k a_{2} r / z\right)}{k a_{2} r / z}\right] \\
& -\left.\left(\pi a_{1}^{2}\right)\left[\frac{2 J_{1}\left(k a_{1} r / z\right)}{k a_{1} r / z}\right]\right|^{2} .
\end{aligned}
$$

The MTF is shown in Fig. 3(b). The maximum resolvable spatial frequency remains constant regardless of obscuration, but large obscuration severely reduces the midspatial frequency contrast. Postdetection processing can restore contrast in the final image provided there is sufficient dynamic range in the detector and no zeros exist in the MTF. ${ }^{10}$

Given an image sensor with limited signal-to-noise ratio (SNR), the reduction in the MTF reduces the

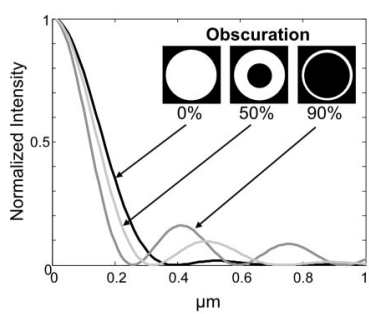

(a)

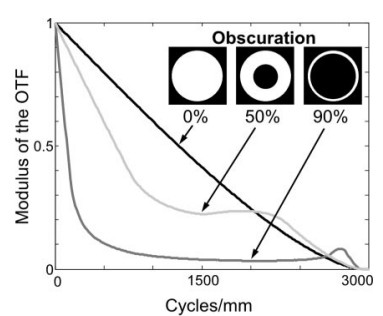

(b)
Fig. 3. Diffraction-limited incoherent (a) PSF and (b) MTF of a $60 \mathrm{~mm}$ circular aperture with different levels of central obscuration (\% OD). 


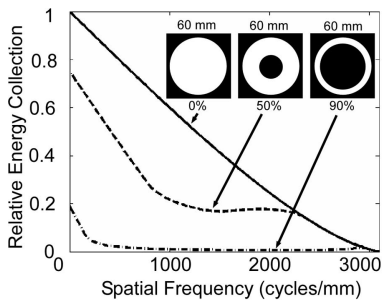

(a) (b)

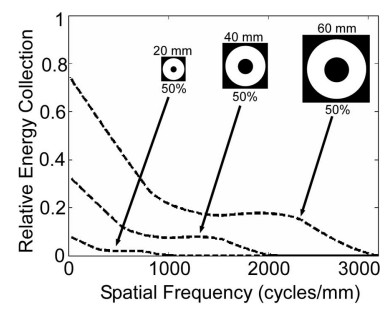

Fig. 4. Relative energy collection versus spatial frequency for various circular apertures. (a) Comparison of different obscurations with fixed OD $(60 \mathrm{~mm})$. (b) Comparison of different diameters with fixed obscuration (50\%). Plots are normalized to the unobscured $60 \mathrm{~mm}$ optic. All the simulations have an EFL of $35 \mathrm{~mm}$.

unprocessed image contrast compared with an unobscured optic. To match the contrast, the diameter of the folded optic must be increased to raise the total signal power to at least the value of the unobscured optic at all spatial frequencies of interest. To directly and more clearly compare obscured and unobscured optics, it is useful to examine the MTF as a representation of relative energy collection as a function of spatial frequency. Consider the definition of the MTF given by Eq. (3) as $^{9}$
Table 1. Size Comparisons to Match Light Collection Aperture Area to an $f / 1.435 \mathrm{~mm}$ Conventional Lens ${ }^{a}$

\begin{tabular}{lccc}
\hline & Track & Diameter & Volume \\
\hline Conventional lens (reference) & 1.00 & 1.00 & 1.00 \\
Fourfold, $\mathrm{CaF}_{2}$ substrate & 0.36 & 1.60 & 0.91 \\
$\quad$ Fourfold, air gap & 0.25 & 1.60 & 0.64 \\
Eightfold, $\mathrm{CaF}_{2}$ substrate & 0.18 & 2.19 & 0.86 \\
Eightfold, air gap & 0.12 & 2.19 & 0.60 \\
\hline
\end{tabular}

${ }^{a}$ Values relative to a conventional lens.

energy as a function of spatial frequency. From these values of track and diameter, we can also estimate the volume of the folded optic for comparison with a conventional imager of the same focal length.

To make this comparison, we consider a conventional high-resolution, compact camera lens with typical attributes: an $f / 1.4$ lens with a $35 \mathrm{~mm}$ focal length. We can model this lens volume as a cylinder with a diameter (open aperture) of $25 \mathrm{~mm}$ and a physical track length of $35 \mathrm{~mm}$. This reference optic will be compared with a simplified folded optic with the same focal length and a form determined by the number of folds and the diameter required to achieve the same optical energy collection. Since reflective systems can

$$
\mathrm{MTF}=\frac{\iint_{\infty} P\left(x+\left(\lambda z_{i} f_{x} / 2\right), y+\left(\lambda z_{i} f_{y} / 2\right)\right) P\left(x-\left(\lambda z_{i} f_{x} / 2\right), y-\left(\lambda z_{i} f_{y} / 2\right)\right) \mathrm{d} x \mathrm{~d} y}{\iint P(x, y) \mathrm{d} x \mathrm{~d} y} .
$$

Here, the definition of the MTF is expressed in its usual form as the autocorrelation of the pupil normalized by the area of the pupil. Removing the normalization factor, or normalizing to a comparison imager, allows for apertures of different sizes and shapes to be directly compared in terms of the apertures diffraction-limited relative energy collection versus spatial frequency. Normalizing to a clear $60 \mathrm{~mm}$ aperture, Fig. 4 illustrates how circular obscured apertures of different diameters and obscurations can be directly compared in terms of energy collection for a fixed focal length.

\section{Volume and Thickness Comparison}

Since the motivation of folded optic systems is size reduction, it is useful to compare the volume, track, and performance of a folded lens compared with a conventional lens of the same effective focal length (EFL). Given the basic form of a folded lens (number of folds and obscuration ratio), we can estimate the total track of the folded optic and the diameter needed to match either the total light collection area or the relative have low $f$ numbers without incurring chromatic dispersion, we can assume all the optical power at the first reflection of the folded optic. In this case, the total physical thickness $T$ is approximately

$$
T \cong \frac{\mathrm{EFL} * n_{s}}{N}
$$

where $n_{s}$ is the refractive index of the internal volume of the folded optic and $N$ is the number of folds in the optical design. For simplicity, no telephoto reduction has been assumed in Eq. (4) or the conventional comparison lens. The results using Eq. (4) for eightfold and fourfold optical designs with calcium fluoride $\left(\mathrm{CaF}_{2}\right)$ and air-gap substrates can be found in Table 1. Here we find a $2.5 \times-8 \times$ reduction in total optical thickness depending on the number of folds and the lens material.

Using these calculated track lengths and assuming an obscuration ratio, we can also estimate the total volume of the folded designs to match the collection aperture area of the conventional lens. Obscuration 


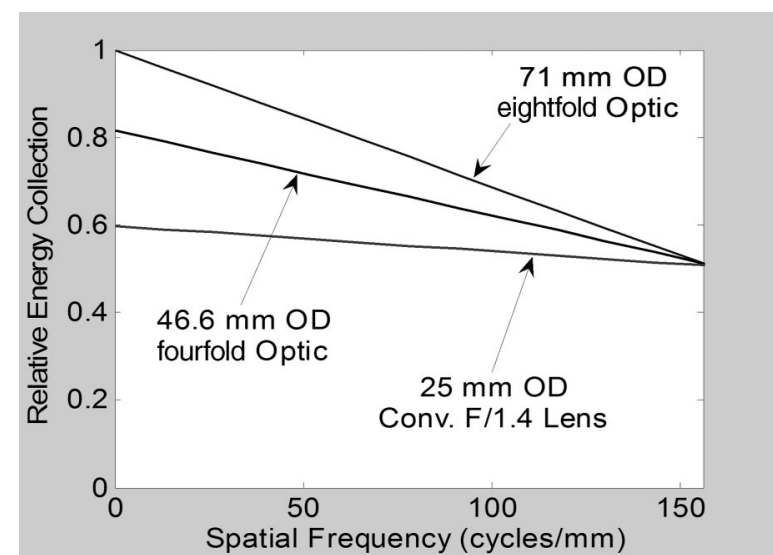

Fig. 5. Relative energy collection versus spatial frequency for eightfold and fourfold designs matched to a $35 \mathrm{~mm} / 1.4$ conventional lens up to 156 cycles/mm.

ratios for the eightfold and fourfold optics must be assumed so we will use values of $89 \%$ and $78 \%$, respectively (values based on functional designs discussed in Section 3 of this paper). The results for the diameter and volume of the folded optics to match the collection aperture area are summarized in Table 1. To match total collection aperture area, these example folded optics each display an increase in diameter but a reduction in both optical track and volume compared to an $f / 1.4$ conventional comparison lens.

In addition to comparing simple collection aperture area, it is useful to match the performance of folded optics in terms of the frequency domain energy collection. This is a much more stringent comparison with highly obscured apertures since the MTF falls off steeply as a function of spatial frequency compared with the relatively flat unobscured conventional lens. The aperture of the folded lens must be scaled up considerably to match or exceed the energy collection of the conventional lens up to a desired cutoff frequency. Choosing a maximum spatial frequency of $156 \mathrm{cycles} / \mathrm{mm}$ (cutoff frequency for $3.2 \mu \mathrm{m}$ pixels), we find that the diameters of the eightfold and fourfold optics must be scaled up $2.84 \times$ and $1.86 \times$, respectively, to match frequency domain energy collection with respect to the conventional reference lens up to sensor cutoff. This is shown in Fig. 5.

Table 2 shows the comparison results for matching the frequency domain energy collection. Here the diameters of the folded optics must be scaled up significantly to match the frequency domain energy collection up to cutoff, which can result in greater volume than the comparison lens. This comparison may be overly strict since the matched folded imagers have a much higher total light collection than the comparison lens.

To this point, we have not included field of view (FOV) in the comparison between folded and conventional optic imaging. The obtainable FOV in folded optic designs is constrained by the inner diameter of the last rear mirror and is in general less than a well-corrected conventional lens with the same aper-
Table 2. Size Comparisons to Match Relative Energy Collection to a $\mathrm{f} / 1.435 \mathrm{~mm}$ Conventional Lens up to $156 \mathrm{cycles} / \mathrm{mm}^{a}$

\begin{tabular}{lccc}
\hline & Track & Diameter & Volume \\
\hline Conventional lens (reference) & 1.00 & 1.00 & 1.00 \\
Fourfold, $\mathrm{CaF}_{2}$ substrate & 0.36 & 1.86 & 1.25 \\
Fourfold, air gap & 0.25 & 1.86 & 0.86 \\
Eightfold, $\mathrm{CaF}_{2}$ substrate & 0.18 & 2.84 & 1.45 \\
Eightfold, air gap & 0.12 & 2.84 & 0.97 \\
\hline
\end{tabular}

${ }^{a}$ Values relative to the conventional lens.

ture diameter. Reference 11 is an analysis of compact multiaperture and folded imaging systems that cites an early conference presentation of this work. The author concluded that folded imagers decrease system track but require multiple imagers to match the full FOV of a conventional imager. Under Haney's assumptions, a $4 \times$ reduction in thickness would require roughly a $2 \times$ increase in total system volume, and greater length reductions would require a much greater volume. This estimate is substantially verified by our specific imager designs. In some applications, the reduction in track length is critical (i.e., in reducing cantilevered mass in tracking mechanics). Otherwise, folded imaging systems are best suited to applications requiring high resolution but only a moderate FOV.

\section{Specific Design Considerations}

\section{A. Folded Optic Design Space}

For our designs, we have been primarily interested in designing thin imagers of $5 \mathrm{~mm}$ total thickness (front surface to sensor) illuminating $1000 \times 1000$ pixels of an Omnivision 3620 CMOS color sensor. This sensor contains $2048 \times 1536$ pixels of $3.18 \mu \mathrm{m}$ pitch and was chosen for its small pixel pitch and large pixel count of which a smaller subset is used.

The number of folds required in a folded optic is a property that depends on the desired focal length of the optic and the desired total thickness. Figure 6 shows a plot of FOV versus equivalent aperture for several different fold designs, all $5 \mathrm{~mm}$ thick, illuminating the Omnivision CMOS sensor. With the exception of the eightfold design, all the designs have aspheric surfaces on front and back-a more difficult fabrication project than the single-sided eightfold

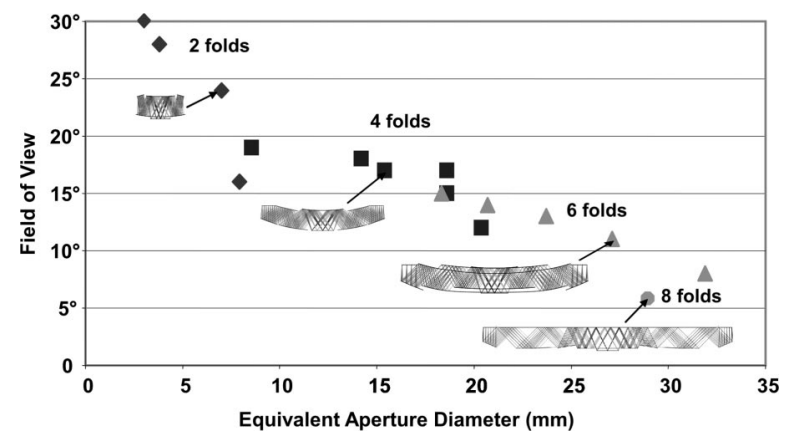

Fig. 6. FOV versus equivalent aperture diameter for several multiple-fold designs. 


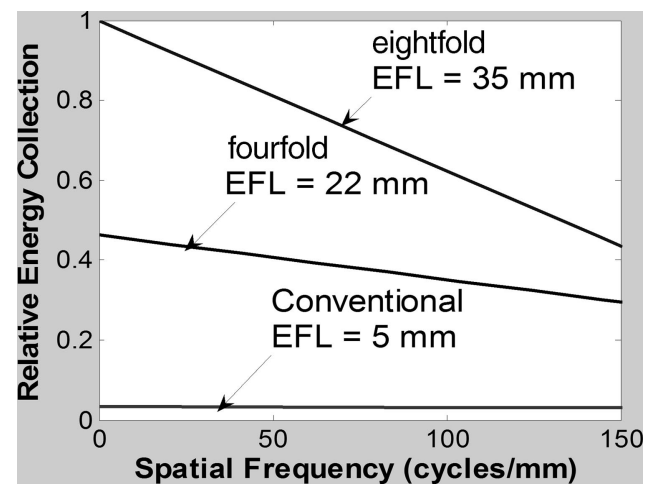

Fig. 7. Diffraction limited relative energy collection versus spatial frequency comparing a conventional fast miniature lens $(f 1$, $\mathrm{EFL}=5 \mathrm{~mm})$, a fourfold design $(\mathrm{EFL}=22 \mathrm{~mm})$ and an eightfold design $(\mathrm{EFL}=35 \mathrm{~mm})$; all of $5 \mathrm{~mm}$ total thickness.

optic. Once optimized, all the designs are shown to lie on a rough line through the chart where the available FOV is reduced as the size of the aperture and the number of folds and corresponding EFL are increased. The designs of a specific number of folds are found to have a limited region where they can be successfully optimized for a given thickness and sensor.

The frequency domain relative energy collection can be used to compare these designs to a conventional miniature camera. Figure 7 shows the diffractionlimited relative energy collection versus spatial frequency for a miniature conventional lens and two folded lens designs, all $\sim 5 \mathrm{~mm}$ total thickness. The conventional lens shown is an $f / 1$ circular aperture with an EFL of $5 \mathrm{~mm}$. The eightfold design shown has an EFL of $35 \mathrm{~mm}$ with an effective aperture diameter of $27 \mathrm{~mm}$ (60 $\mathrm{mm}$ OD, 90\% obscured); and the fourfold design has an EFL of $22 \mathrm{~mm}$ with an effective aperture diameter of $18.5 \mathrm{~mm}$ (30 mm OD, 79\% obscured). These values of EFL and aperture size for the folded optics were taken from working designs for comparison. As shown in Fig. 7, both of these folded optic designs have much higher energy collection and resolution than a conventional miniature lens of the same thickness.

\section{B. Implementation Challenges}

The most significant challenges associated with annular folded optics are fabrication tolerances, depth of field and/or focus, stray light suppression, and optical efficiency. Fabrication tolerances and depth of field and/or focus are for the most part defocus related problems that can be significantly improved through the use of wavefront coding. If the folded optic is fabricated from one piece of material, a thickness error in the fabrication introduces concatenated piston error in the optical path. A refocus of the image plane compensates for the error, but this remains the most severe tolerance. On the other hand, centration tolerances can be effectively eliminated by making the optic planoaspheric where all the powered surfaces reside on one side of the optic, and the optic can be diamond turned without rechucking the substrate.
The narrow depth of field associated with folded optics is attributable to the high NA of the design. However, this property is not unique to folded optics since all high-quality imagers suffer from the same trade-off between resolution (NA) and depth of field and/or focus.

Stray light arises in folded optics when light travels an unintended path through the optic and reaches the sensor as noise. Stray light is commonly reduced with baffles in astronomical telescopes, and the same approach works with multiple-fold optics. With folded optics the regions between concentric reflectors can be cut into baffles and made absorbing to help reduce stray light. Stray light suppression is one of the most important challenges with highly folded imagers and necessary for their use in situations where bright stray light sources, such as the Sun, are present. In such situations, more advanced baffle geometries and angle selective dielectric coatings will be useful to help control washout caused by stray light.

Finally, significant optical attenuation can be caused by multiple reflection losses and by vignetting attributable to the specific sensor geometry. Pixel vignetting is a term used to describe losses in CMOS image sensors because of shadowing from the metal interconnect layers that surround the light-sensitive area. Microlenses are typically used to help focus light onto the sensor, but large angle rays can be blocked, ${ }^{12}$ making the sensors incompatible with the low $f$ number of many injection molded aspheric lenses. This effect is increasingly problematic as the pixel pitch decreases. For a $3 \mu \mathrm{m}$ pitch, the interconnect layer height is greater than the width of the active area. With folded optics, the NA is large, and all the light is incident at large angles. The microlenses can be omitted or index matched to reduce their effect, ${ }^{13}$ but even so, approximately half of the incident light can be blocked by the interconnect layer. A near-term solution is to use CMOS processing with copper rather than aluminum interconnects, which increases conductivity and allows for thinner and narrower traces. There are also development efforts to improve the angle performance of CMOS sensors by wafer thinning and backside illumination. ${ }^{14,15}$

\section{Air-Gap Folded Designs}

We conclude this section with mention of two advantages unique to air-gap folded designs, where two reflective surfaces surround a hollow cavity. When a folded optic is cut from a substrate such as glass or plastic, some residual chromatic dispersion will be present owing to the refraction of light as it enters the optical element, even if the first surface is flat. Airgap versions of the optic eliminate this refraction, yielding an all-reflective imager with zero chromatic aberration. A thin cover sheet used to protect the optical surfaces does not introduce angle dispersion and does not cause significant chromatic aberration. Hollow folded optics will be useful for inexpensive, lightweight IR imagers and may lead to convenient designs for hyperspectral imagers. 
The second interesting property of a folded air-gap imager is squeeze focus. In the same way that substrate thickness errors introduce concatenated piston error in the tolerance analysis, a small adjustment of the distance between the front reflector and the backreflector allows for large changes in the focus of the optic. This sensitivity can be advantageous when compared with the limited amount of refocus possible with simple back focal length adjustment. Folded optics with refractive interiors can also be squeeze focused by fabricating the front reflector and backreflector on separate substrates, and using antireflection-coated or index-matched flat surfaces between the substrates to allow for a variable gap.

\section{Eightfold Prototype}

\section{A. Eightfold Design}

We selected the eightfold plano-aspheric design for fabrication and testing. The goal was a visible light

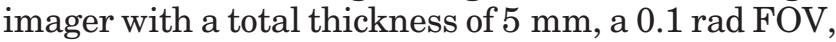
$0.1 \mathrm{mrad}$ resolution, and $>25 \mathrm{~mm}$ effective aperture to illuminate $1000 \times 1000$ pixels of an Omnivision 3620 CMOS color image sensor.

To achieve these specifications, the eightfold optic shown in Fig. 8(a) was optimized by using a solid $\mathrm{CaF}_{2}$ substrate. $\mathrm{CaF}_{2}$ was chosen for its rigidity, low dispersion, and especially for its compatibility with single-point diamond turning technology. The optic has a $60 \mathrm{~mm}$ OD, $53 \mathrm{~mm}$ inner obscuration, $5 \mathrm{~mm}$ thickness, and was designed for a $2.5 \mathrm{~m}$ object distance to facilitate laboratory testing. Light is focused onto the sensor by four concentric aspheric reflectors on the back side of the optic while the front side remains planar to simplify fabrication. Index-matching gel is used between the final transmissive surface of the imager and the sensor to index match the microlenses on the sensor.

The imager achieves an effective aperture diameter of $27 \mathrm{~mm}$, a NA of 0.7 , a FOV of $0.12 \mathrm{rad}\left(6.7^{\circ}\right)$, and a $38 \mathrm{~mm}$ EFL. Resolution is limited to $1280 \times 960$ pixels of the Omnivision sensor. The simulated monochromatic MTF for this optic is shown in Fig. 8(b). This MTF figure shows diffraction-limited monochromatic performance up to and beyond the Nyquist frequency of the sensor sampling. The geometric spot diagrams for the monochromatic and broad-spectrum cases are shown in Figs. 8(c) and 8(d), respectively. As shown in the broad-spectrum figure, the optic displays approximately $8 \mu \mathrm{m}$ of lateral color $( \pm 1$ pixel at full field) aberration because of dispersion at the entrance pupil. This color aberration can be corrected with postdetection processing [remapping of the redgreen-blue (RGB) planes].

With a NA of 0.7 and $\sim 90 \%$ obscured aperture, the depth of focus and depth of field become considerably narrow. The depth of focus for this design is approximately $10 \mu \mathrm{m}$, corresponding to a depth of field of only $24 \mathrm{~mm}$ at the $2.5 \mathrm{~m}$ conjugate. This narrow

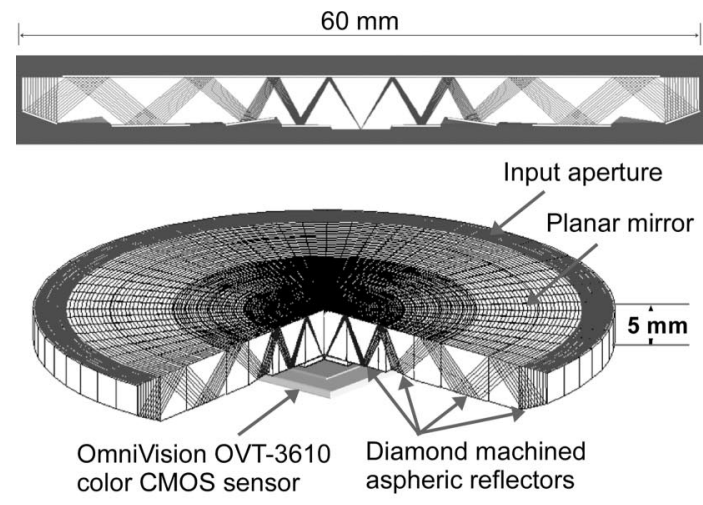

(a)

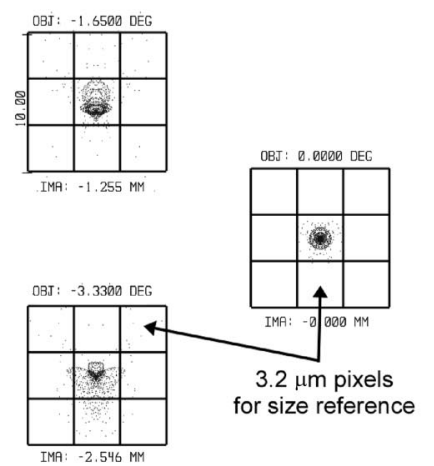

(c)

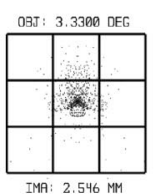

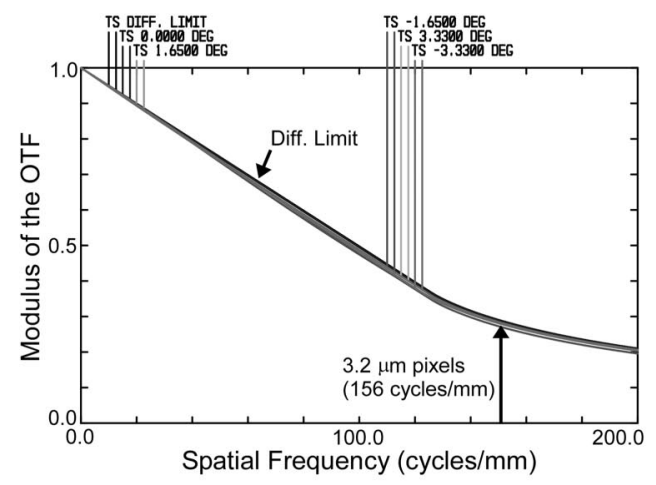

(b)
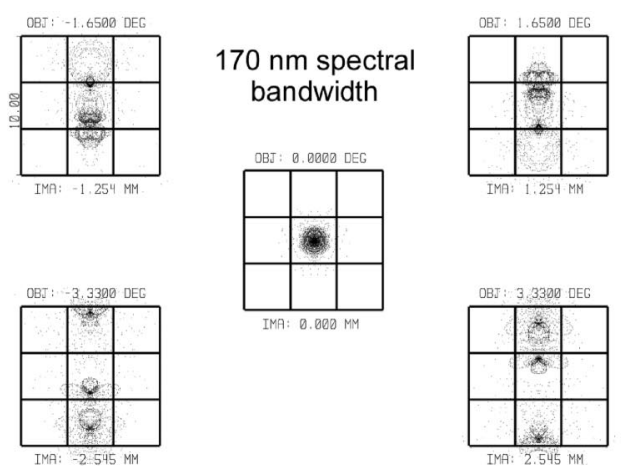

(d)

Fig. 8. (a) Eightfold imager in $\mathrm{CaF}_{2}$ schematic, (b) calculated monochromatic MTF, (c) simulated monochromatic (588 nm) geometric spot diagram, and (d) simulated broad-spectrum $(486,588,656 \mathrm{~nm})$ geometric spot diagram showing $\sim 8 \mu \mathrm{m}$ lateral color ( \pm 1 pixel at field stop). 


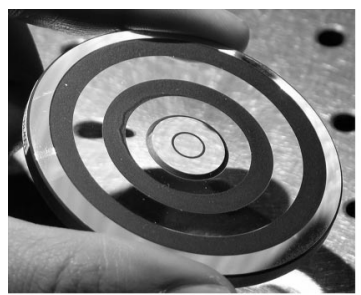

(a)

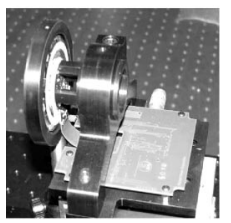

(d)

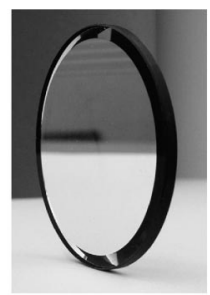

(b)

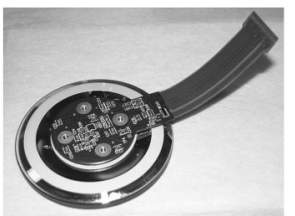

(e)

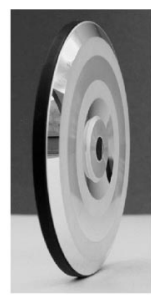

(c)

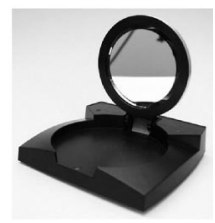

(f)
Fig. 9. Camera prototype fabrication and integration. (a) Diamond turned optic before coating, (b) silver-coated front surface with annular entrance pupil, (c) coated back surface, (d) active alignment of CMOS sensor, (e) fully functional fixed-focus camera, and (f) fixed-focus camera and electronics packaged in a plastic enclosure.

depth of field improves considerably with larger conjugates, but will be a limitation for short object-range applications. Wavefront coding may be employed to improve the depth of field in high-NA-fold designs such as the one described here.

\section{B. Fabrication and Integration}

We had the eightfold design diamond turned in $\mathrm{CaF}_{2}$ by Fresnel Technologies, shown in Fig. 9(a). In between the concentric aspheric surfaces of the back side, black baffles were painted onto the optic to provide a simple method of stray light blocking in the optical path. Once the fabrication was machined, IST Optics completed it by coating the substrate with patterned silver reflectors as shown in Figs. 9(b) and 9(c).

The surface metrology of such a multiple-zone and large-area aspheric optic is difficult. Ideally, a large-area computer generated hologram $(\mathrm{CGH})$ could be fabricated to generate a null wavefront for an interferometric test. Without this test surface, we used a less accurate examination of the aspheric side by using a $50 \times, 0.42 \mathrm{NA}$ microscope objective along with computer-controlled positioning to compare relative surface coordinates to the specified optical design. This measurement had $\sim 5 \mu \mathrm{m}$ absolute positioning accuracy, allowing us to verify gross element thickness and zonal surface shift but not the surface figures of the aspheric reflectors. The annular surface shifts and the total element thickness were verified to be within the specified $10 \mu \mathrm{m}$ tolerances. The surface figure of sections of the zone aspheric reflector were verified by using a large-magnification white-light interferometer (ADE Phase-shift MicroXAM), which showed an average polished roughness of $5 \mathrm{~nm} \mathrm{rms}$ with peaks to $50 \mathrm{~nm}$ (toolmarks) on the aspheric side of the optic. The surface of the nominally planar front reflector was

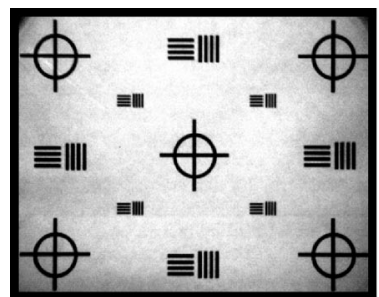

(a)

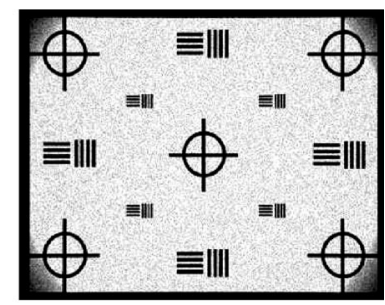

(b)
Fig. 10. (a) Image taken with eightfold camera prototype and (b) Zemax predicted image.

measurable by using a Michelson interferometer that revealed an approximately spherical curvature across the surface with $500 \mathrm{~nm}$ of sag at the center of the bowed front surface. This value of sag exceeded our tolerance spec of $0.1 \lambda$ at $546 \mathrm{~nm}$, but ray tracing indicated that refocus of the back focal distance by $10 \mu \mathrm{m}$ reduced the effect of the aberration to tolerable levels.

We aligned and integrated the optic and sensor with index-matching gel between them using a fiveaxis stage and the mounting assembly shown in Fig. 9 (d). Once optically aligned to an object at $2.5 \mathrm{~m}$, the sensor and flex circuit were attached to the back of the optic [Fig. 9(e)] with an area of rigid UV-cured epoxy later surrounded by a flexible UV-cured epoxy for strain relief. The optic was mounted into the black plastic case with the camera and the universal serial bus (USB) driver circuitry [Fig. 9(f)] as a fixed focus prototype camera.

\section{Image Quality-Comparisons and Performance}

Figure 10(a) shows the image of a resolution chart taken with the fixed focus camera prototype at $2.5 \mathrm{~m}$. The image on the right [Fig. 10(b)] is a Zemax geometric image simulation of the same object through the eightfold optic. Visually, resolution and uniformity appear to be nearly identical. Vignetting can be seen in the corners of both images where regions of the object extend outside of a $1280 \times 960$ pixel FOV.

To make a comparison test of the eightfold multifold camera with a conventional camera, we set up a refractive zoom lens with the same magnification, aperture size, Omnivision sensor, and software. Placed side by side [Fig. 11(a)], the two cameras image a series of resolution charts [Fig. 11(b)] spaced $7 \mathrm{~cm}$ apart to show depth of field effects. Images from both cameras can be seen in Figs. 11(c) and 11(d) corresponding to the conventional camera and folded camera, respectively. At best focus, the resolution, color, and image quality are similar between the two cameras. Out of focus, the folded lens is easily identified with a more pronounced blur owing to the narrow depth of field.

To quantitatively compare the performance of the two cameras, we measured the MTF by using a sinusoidal modulation chart of varying spatial frequency. Figure 12 shows the two measured MTFs for the folded and conventional cameras, illustrating the 


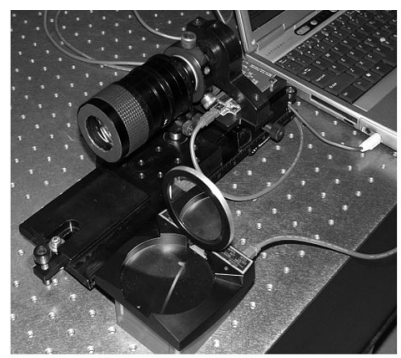

(a)

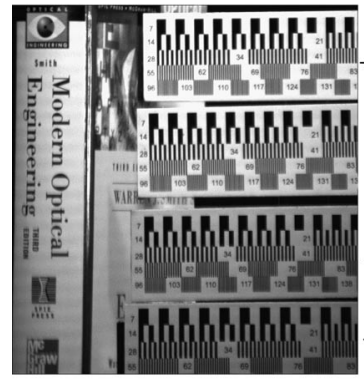

(c)

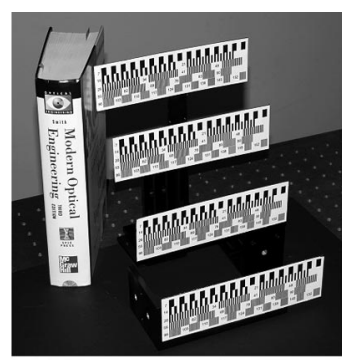

(b)

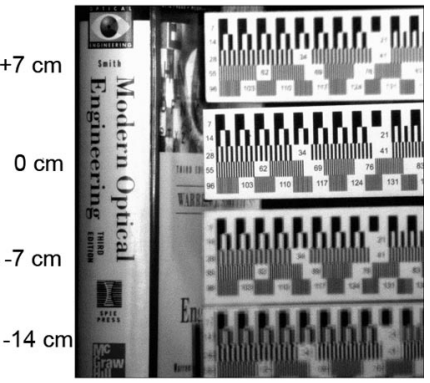

(d)
Fig. 11. Conventional and eightfold image comparison. The dual camera setup (a) is used to image (b) the staggered resolution charts. (c) and (d) show the images taken with the conventional camera and eightfold camera, respectively.

similarity in resolution and image quality between the two cameras at best focus.

The quality between the two imagers becomes more disparate at lower light levels. With approximately $10 \times$ less light, noise in the folded optic's image is revealed. To obtain visually comparable images, it was found that the folded optic required 600 $\mathrm{ms}$ exposure to accomplish what the conventional camera images in $\sim 4 \mathrm{~ms}$. This difference in efficiency comes from preventable losses in the reflective coating of the folded optic and unsuitable sensor geometry. Reflective losses can be improved by using a highly efficient dielectric reflector rather than the currently used silver reflector. Reflecting $8 \times$, the silver reflector passes only $12 \%$ of the light compared

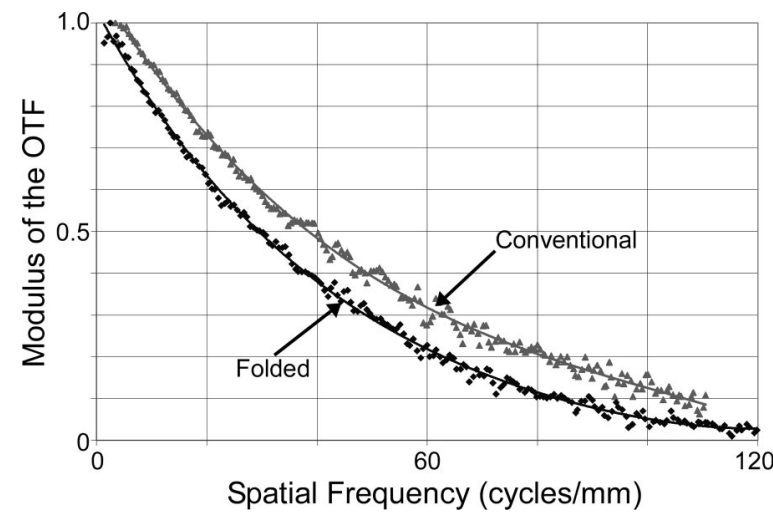

Fig. 12. Measured in-focus MTF (optics + sensor) comparison of the eightfold imager and the conventional comparison imager using identical CMOS image sensors. with a properly designed dielectric reflector with approximately $80 \%$ achievable efficiency. Our first measurement of an imager with a dielectric mirror showed approximately $43 \%$ total efficiency including surface transmission and eight mirror reflections.

Pixel vignetting from the sensor's interconnect layer can be reduced by switching to a more suitable sensor structure. Although the exact tunnel height specification for the Omnivision sensor is not available, we can approximate a large amount of loss $(\sim 10 \mathrm{~dB})$ in our camera with a typical $3.6 \mu \mathrm{m}$ interconnect layer and our range of incidence angles. Switching to a different sensor format with reduced interconnect layer thickness or rear illuminated back-thinned sensors will significantly improve SNR in folded imagers such as the prototype fabricated here.

\section{Thermal Testing}

Finally, we tested the thermal operation range of the camera prototype by imaging the depth of field scene of Fig. 11 with the camera in a vacuum oven looking out through a window. Images were taken for comparison at $23{ }^{\circ} \mathrm{C}$, after $90 \mathrm{~min}$ at $60{ }^{\circ} \mathrm{C}$, and after cool down to $23^{\circ} \mathrm{C}$. The effect of heating on the folded optical element was an $\sim 1 \%$ change in focal position owing to thermal expansion in the $\mathrm{CaF}_{2}$ substrate. Heating also caused strong color variation and increased noise in the CMOS image sensor. At high temperatures, the raw images from the sensor were blueshifted with reduced image contrast because of the elevated noise. Finally, and of least consequence, the elevated temperature introduced a mechanical tilt in the hinge of the plastic package giving a slight tilt to the captured image. There was, however, no apparent damage to the camera from the test. The resolution, color balance, and focus were all restored after the imager was cooled down.

\section{Conclusions}

We have extended the well-known Cassegrain telescope to the larger class of annular folded optic imagers, considering the fundamental physical optical effects of highly obscured apertures. We have shown that the imager size can be scaled by using a normalized modulation transfer function to match the spatial frequency response of clear aperture imagers and that for optical imagers with moderate field of view requirements, highly folded imagers provide an effective way to substantially reduce optical track and, potentially, total system weight and volume.

After looking at designs with two to eight folds, we selected an $89 \%$ obscured eightfold design for experimental fabrication and test. We used an optic produced by conventional diamond turning to fabricate a color imager with $1280 \times 960$ pixel resolution (over a $0.12 \mathrm{rad}$ FOV) where a $38 \mathrm{~mm}$ effective focal length is folded into a total optical track (lens face to image sensor) of just $5 \mathrm{~mm}$. The self-contained imager, integrated with a USB interface to a PC, performed close to the optical design performance under laboratory and elevated temperature conditions. 
Looking ahead, such annular folded optics have potential for compact imagers where long focal lengths must be contained within a limited track. The allreflective design is particularly suitable for IR telescopes, offering improvements over relatively bulky conventional refractives made of expensive materials.

This work was performed as part of a larger program in multidomain optimization and benefited from many technical discussions with Mark Neifeld (University of Arizona), Shaya Fainman, Uriel Levy, and Hyo-Chang Kim (University of California San Diego), Joel Rutkowski and Inga Tamayo (CDM Optics), and Ravi Athale and Dennis Healy [Defense Advanced Research Projects Agency (DARPA)]. We acknowledge the fabrication services provided by Fresnel Technologies and IST Optics. This research was supported by DARPA via the Multiple Optical Non-Redundant Aperture Generalized Sensors program, grant HR0011-04-I-0045, and by the Natural Sciences and Engineering Research Council of Canada (NSERC).

\section{References}

1. M. Laikin, Lens Design (Dekker, 2001), Chap. 15.

2. V. Draganov and D. G. James, "Compact telescope for freespace communications," in Current Developments in Lens Design and Optical Engineering III, R. E. Fischer, W. J. Smith, and R. B. Johnson, eds., Proc. SPIE 4767, 151-158 (2002).

3. E. J. Tremblay, J. Rutkowski, I. Tamayo, R. A. Stack, R. L. Morrison, D. Combs, J. Mader, H.-C. Kim, U. Levy, Y. Fainman, and J. E. Ford, "Ultrathin folded imager," in OSA
Topical Meeting on Computational Optical Sensing and Imaging (Optical Society of America, 2005).

4. W. Kordonski, A. Shorey, and A. Sekeres, "New magnetically assisted finishing method: material removal with magnetorheological fluid jet," in Optical Manufacturing and Testing, V. H. P. Stahl, ed., Proc. SPIE 5180, 107-114 (2003).

5. E. R. Dowski and W. T. Cathey, "Extended depth of field through wavefront coding," Appl. Opt. 34, 1859-1866 (1995).

6. E. L. O'Neill, "Transfer function for an annular aperture," J. Opt. Soc. Am. 46, 285-288 (1956).

7. V. N. Mahajan, "Imaging with obscured pupils," Opt. Lett. 1, 128-129 (1977).

8. J. E. Harvey and C. Ftaclas, "Diffraction effects of telescope secondary mirror spiders on various image-quality criteria," Appl. Opt. 34, 6337-6349 (1995).

9. J. W. Goodman, Introduction to Fourier Optics (McGraw-Hill, 1968), pp. 61-113.

10. A. Rosenfeld and A. C. Kak, Digital Picture Processing (Academic, 1982), Vol. 1, pp. 276-281.

11. M. W. Haney, "Performance scaling in flat imagers," Appl. Opt. 45, 2901-2910 (2006).

12. P. B. Catrysse and B. A. Wandell, "Optical efficiency of image sensor pixels,” J. Opt. Soc. Am. A 19, 1610-1620 (2002).

13. G. Agranov, V. Berezin, and R. H. Tsai, "Cross talk and microlens study in a color CMOS image sensor," IEEE Trans. Electron Devices 50, 4-11 (2003).

14. J. Adkisson, J. Gambino, T. Hoague, M. Jaffe, J. Kyan, R. Leidy, D. McGrath, R. Rassel, D. Sackett, and C. V. Stancampiano, "Optimization of $\mathrm{Cu}$ interconnect layers for $2.7 \mu \mathrm{m}$ pixel image sensor technology: fabrication, modeling, and optical results." in Proceedings of 2005 IEEE Workshop on CCD and Advanced Image Sensors (IEEE, 2005), pp. 1-4.

15. J. Janesick, "Lux transfer: complimentary metal oxide semiconductors versus charge-coupled devices," Opt. Eng. 41, 1203-1215 (2002). 